\title{
Development Process For Applications Of Automated Planning For Satellites Control
}

\author{
Charles-Edouard Winandy ${ }^{1}$ and Maurício Gonçalves Vieira Ferreira. ${ }^{2}$ \\ The Brazilian National Institute for Space Research - INPE, São José dos Campos, SP, Brazil
}

\begin{abstract}
The increasing number of satellites which are in orbit around our planet, reflects the growing demand for controllers and control centers for satellites. Although this needs are supplied year after year by new controllers, with some difficulties on some countries, it will soon be a time on which this kind of monitoring will be overtaken by the growing number of satellites, if we don't have an automated monitoring plan. It is important to have, before thinking of implementing a planner involving AI (Artificial Intelligence) or even before create a knowledge base equipped with meta-models, a development process divided in very well defined steps where each step has a target objective to reach and artifacts to generate, using for this modeling techniques used in commercial systems, such as UML. A development process for an automated planning system, capable to decide by itself, based on the satellite components status, the earth based stations and its orbit frequency, must be discussed as soon as possible, in order to grant the future of our capability to safely control all of our satellites. This paper object is to show a software development process when a satellite mission planning is needed to reach the above goals.
\end{abstract}

$\begin{array}{ll}\text { AI } & =\text { Artificial Intelligence } \\ \text { APS and MVP } & =\text { Adaptive Problem Solver and Multimission VICAR Planner } \\ C C S & =\text { Satellites Control Center } \\ E U P & =\text { Enterprise Unified Process } \\ F O P & =\text { Flight Operations Plan } \\ I N P E & =\text { Brazilian National Institute for Space Research } \\ J P L & =\text { Jet Propulsion Laboratory } \\ U M L & =\text { Unified Modeling Language }\end{array}$

\section{Introduction}

$\mathrm{T}$

HE major concern of the INPE CCS is to monitor and control any Brazilian satellite, as well as the foreign partners satellites under his responsibility, in a cohesive and safe way, with the objective to take the very advantages of the allocated equipment and resources.

Due to the vast Brazilian territory and the preoccupation in accompanying the annual deforestation of the Amazonian rain Forest, the satellites started to be very important tools for the country, despite the limited government allowance intended for the sector. On account of this budgetary shortage, the computational and human resources optimization become essential for the INPE. Through the automation and use of AI, the INPE hopes to lower costs without interrupting the growing number of satellites to control in the near years.

Many things can be done and even be in this matter, but the lack of a definite and focused development process in this field of INPE interest has been a problem, in order to grant the ambitious aimed results. The standardization and necessary organization for handling the generated products, as well as the necessity of integrating the produced materials to a group of different persons with different profiles (the researcher's board is formed by students, teachers, public officials and probationers), demands a model of development process to be followed.

This model would be mainly, but not exclusively, used in the preparation of the FOP (Flight Operations Plan) automation, essential for the satellites control. The human intervention is today necessary to the FOP preparation, but, to reach a high automation is intended in the future, which will be only possible to be obtained in a long term delay.

\footnotetext{
${ }^{1}$ Systems Analyst, Stefanini IT Solutions, cwinandy@stefanini.com.

${ }^{2}$ Researcher, Satellites Control Center of INPE, mauricio@ccs.inpe.br, AIAA Member.
}

1

American Institute of Aeronautics and Astronautics 
Just because of treating a continuous process that will pass in the hands of many individuals, and because of always having a group of persons with different profiles, we thought on using the UML, describing the products generated under the adopted standards, for each of the model stages.

\section{PRODESEA}

This process, created to be followed during the automated software development by INPE CCS, was named PRODESEA. This name is an acronym for the Brazilian Portuguese "Automated Space Systems Development Process" translation.

Divided in 6 stages, the PRODESEA ends with the FOP generation, since his aim ends before the execution of the plan created with AI. The 6 sequential stages which compose the PRODESEA, as well as some of its substages follows:

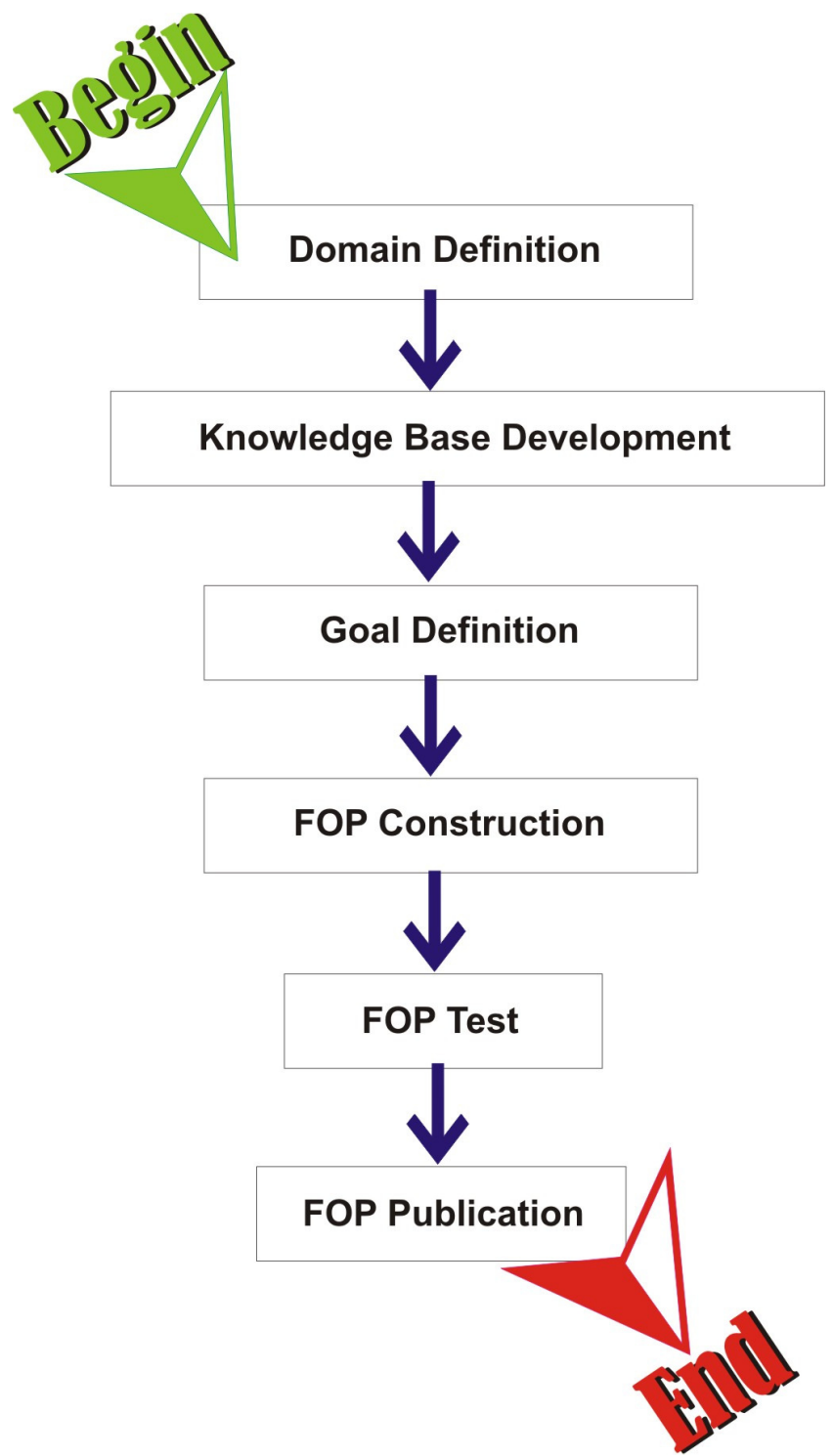

Figure 1. The PRODESEA overview

The process is still in construction, but its first two stages were already completely developed and they will be discussed in this paper. Four other stages will be described without their substages.

2

American Institute of Aeronautics and Astronautics 


\section{A. Domain Definition}

One of the first activities involving a development process, is the definition of the domain problem, also known as application domain. The problem domain is basically a textual description of the problem itself inside its environment. The domain analysis is an attempt to spot the objects, operations and relationships between what experts in a determined domain define as important for the preparation of the solution ${ }^{1}$.

Mostly, an approach first happens at a higher level, with the preparation of a natural language text, then, to a lower level of abstraction, describing the problem in a more technical language, such as algorithms and diagrams. There are some modeling techniques, or diagram notations, like the UML, which can be used for the construction of the domain problem.

A modeling language should target the domain concepts for the problem and not detail it to implement its solution. It should be formal and useful, not only to the domain experts, but also to all development tasks, making executable code, documentation and some types of development tests possible, as well as giving the needed support for the stand alone tools, which should allow the experts to organize the frameworks ${ }^{2}$.

Nevertheless, to reach this level of modeling, a good business environment knowledge is needed. This is why the first designated substage of the PRODESEA Domain Definition stage was the Business Analysis, as shown on figure 2:

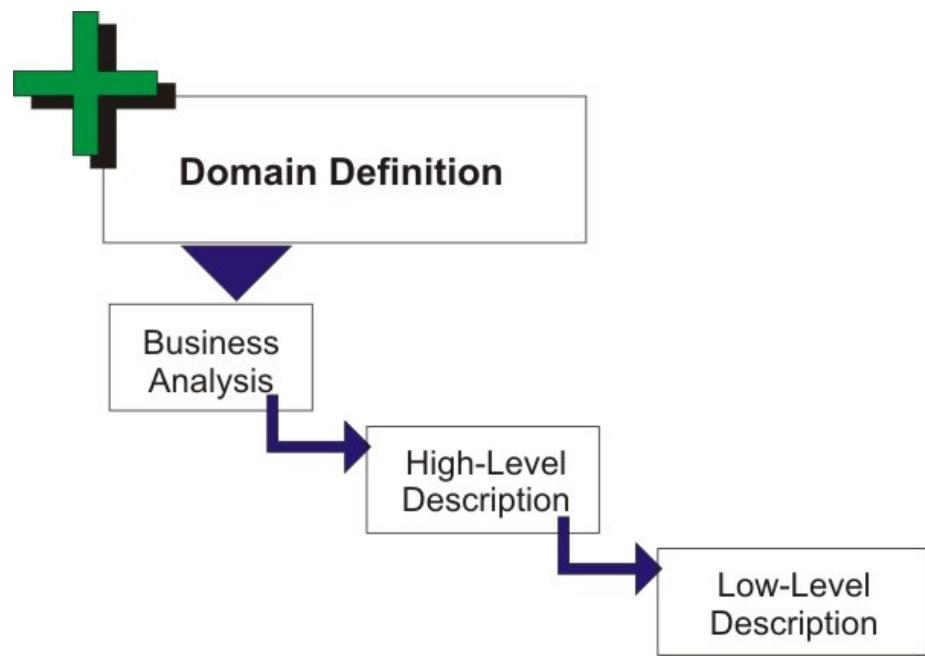

Figure 2. The Business Analysis as primordial stage of the Domain Definition

The Business Analysis was described as a sub-process formed by four stages, the first three picked from the EUP - Enterprise Unified Process. In PRODESEA, this is viewable on Figure 3:

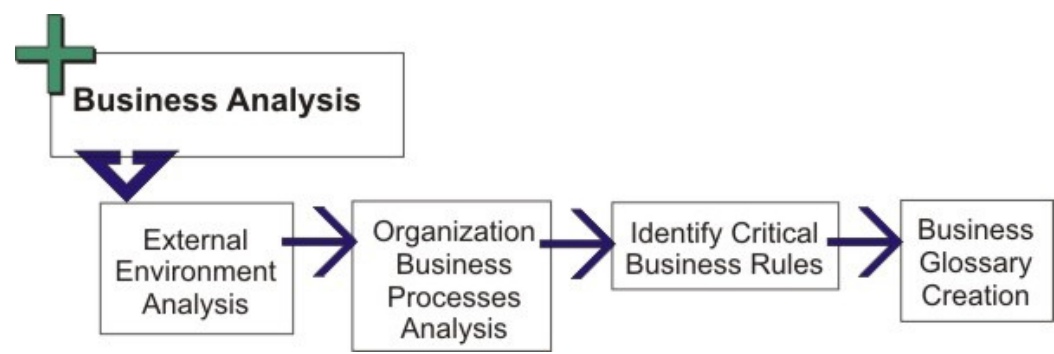

Figure 3. The Business Analysis splitted into four stages

The first step is understanding the External Environment, the context and environment in which the business was built. Next, it is needed to analyse the Business Processes Organization, to spot on the Critical Business Rules in a superficial level in first instance, without detailing too much the rules at this substage of the process. These critical rules will persist along the whole process development, being checkable through tests on its final stages ${ }^{3}$.

The last stage of the Business Analysis is to build a Business Glossary with the most used terms and definitions by the business environment, which in our case is the space area. This document is important for the communication 
between the project Stakeholders, so that all the working persons speaks the same language during the whole development process.

The second substage of the Domain Definition stage is creating a textual description of the domain problem. Despite of working on top of a general vision of the problem and treat only the domain problem abstraction in high level, coming after the stage of the Business Analysis, the descriptive document created will be written by someone having a good business knowledge, respecting the Business Glossary.

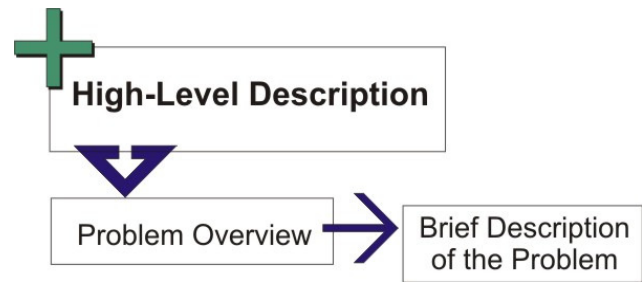

Figure 4. High level of abstraction description of the problem

Once a Brief Description of the Problem has been written, an abstracted approach in a lower level is possible, and so more technical, by using the modeling techniques previously mentioned, as proposed on Figure 5:

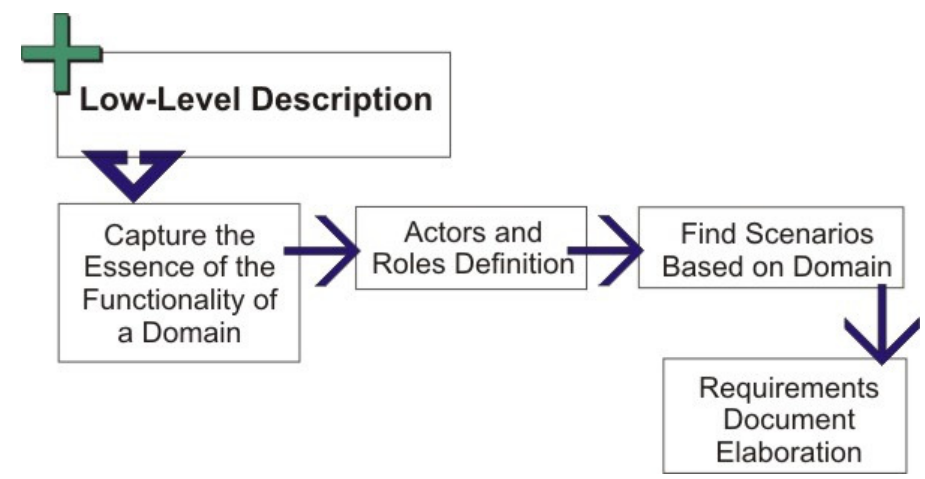

Figure 5. Lower level of abstraction description of the problem

Beginning with the capture of the essential domain functions and passing through the actors and roles definition, it is possible to prepare all sceneries for the problem and its solutions on the focused domain. The functions become cases and the actors interacts with these functions in the defined sceneries. Finally, with the help of Engineering Requisites, a more technical document is written, on which the system or the solution will react to the domain problem.

\section{B. Knowledge Base Development}

With the domain problem well studied, it is possible to go to the Knowledge Base Development stage, since it is only possible to build a Specialized System after a good understanding of the domain problem and its relationships ${ }^{4}$.

The Knowledge Engineering main objective is to provide guidelines to a Specialized System project. The Knowledge Engineering main task is to build the Knowledge Base, which will be used in a decision process 5 .

To build the Knowledge Base, PRODESEA followed three stages, usually used in the acquisition of knowledge according to Elizabeth Cordingley (1989), which are respectively: Definition or Initial Analysis (the decision of which knowledge is necessary); the Knowledge Elicitation (acquisition and interpretation of knowledge, predominantly acquired from experts) and the Knowledge Representation (knowledge coding to the system internal language $)^{6}$. 


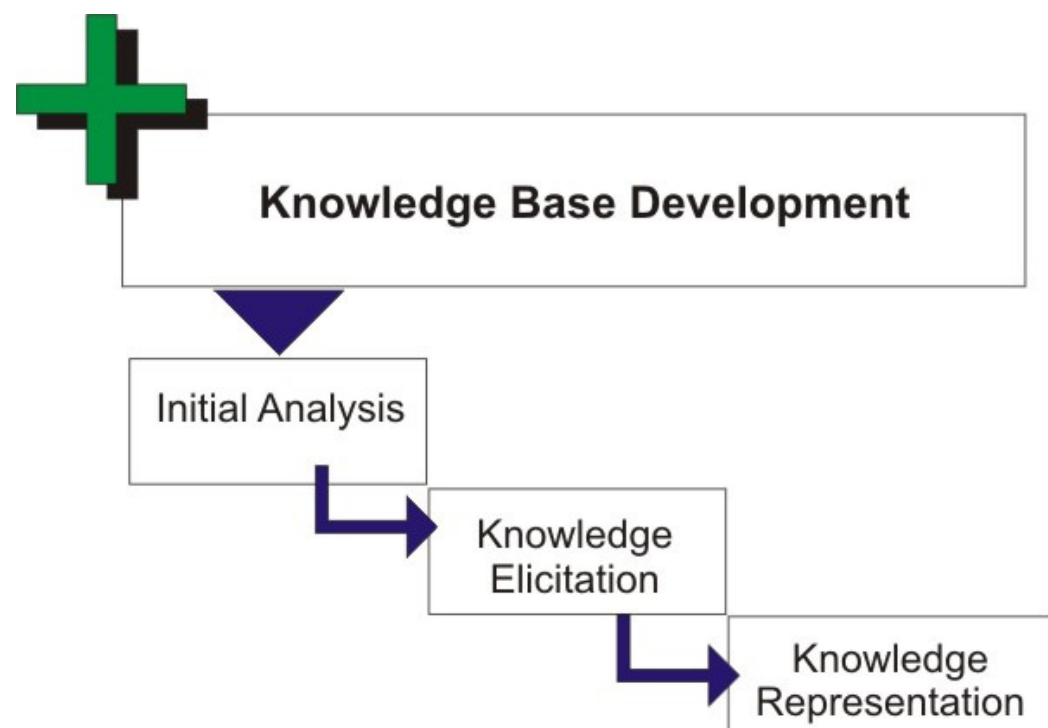

Figure 6. Acquisition and representation of the knowledge for the PRODESEA second stage

Each one of the three substages shown in the Figure 6 uses part of Martin's and Oxman (1988) algorithm, which describes a technique for the knowledge acquisition ${ }^{7}$ for Specialized Systems and can be checked in Figure 7 and read in Table 1 as follows:

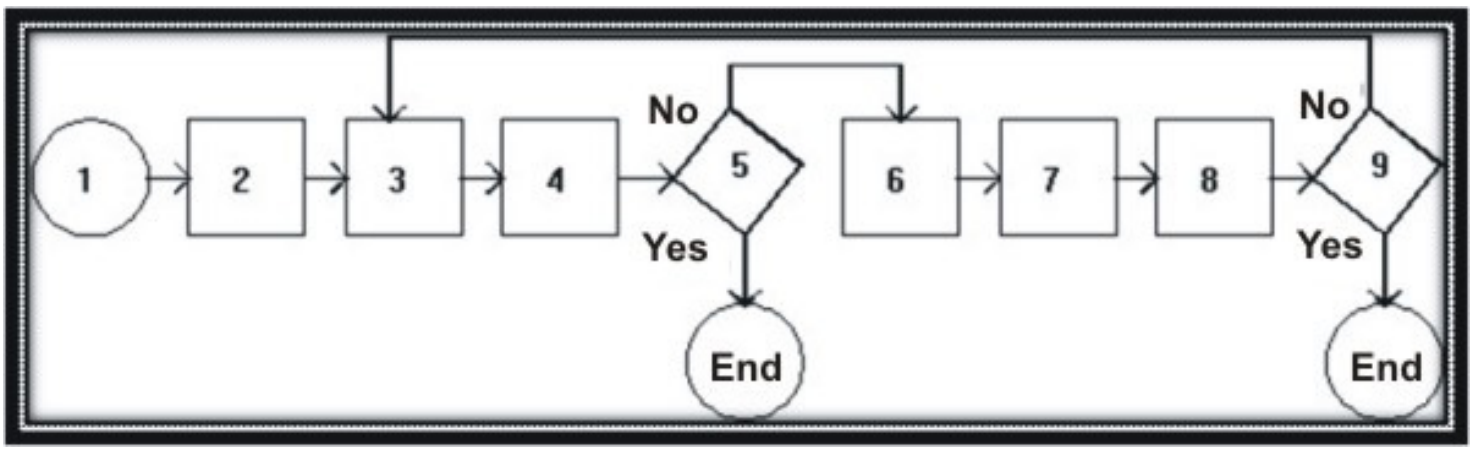

Figure 7. Routine proposed by Martin and Oxman for knowledge acquisition.

\begin{tabular}{|l||}
\hline 1. Acquire initial knowledge of the domain; \\
\hline 2. Make a prototype of the knowledge and insert it in a SHELL for testing; \\
\hline 3. Provide a task example to the prototype; \\
\hline 4. Let an expert observe the system; \\
\hline 5. If the Knowledge Base is reasonably complete, exit; Otherwise, jump to step 6 and continue the process; \\
\hline 6. Let the expert infer what is necessary to the Knowledge Base; \\
\hline 7. Acquire the necessary extra knowledge; \\
\hline 8. Add the necessary knowledge to the Knowledge Base; \\
\hline 9. If the Knowledge Base is reasonably complete, exit; Otherwise, jump back to step 3. \\
\hline \hline
\end{tabular}

Table 1. Routine proposed by Martin and Oxman for knowledge acquisition.

5

American Institute of Aeronautics and Astronautics 
The first substage of Knowledge Base Development from PRODESEA, labeled Initial Analysis, includes the first four steps of the Martin and Oxman algorithm, treating pure knowledge acquisition, as it can be observed in the Figure 8:

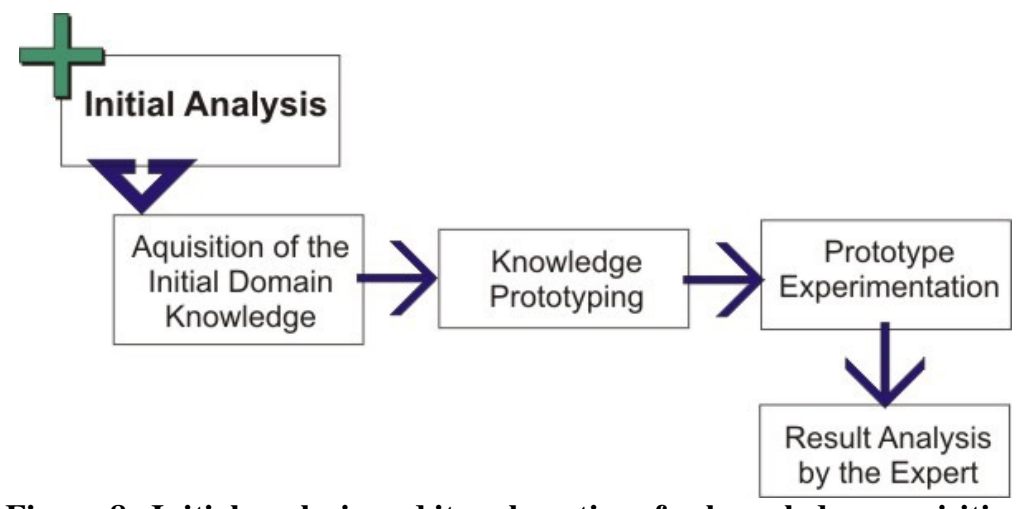

Figure 8. Initial analysis and its subroutines for knowledge acquisition

Basically, from an initial knowledge of the domain, a prototype is built and tested. The results will be analyzed by an expert, then, the achievement and interpretation of the knowledge predominantly come from experts through the substage Knowledge Elicitation takes place, according to Figure 9:

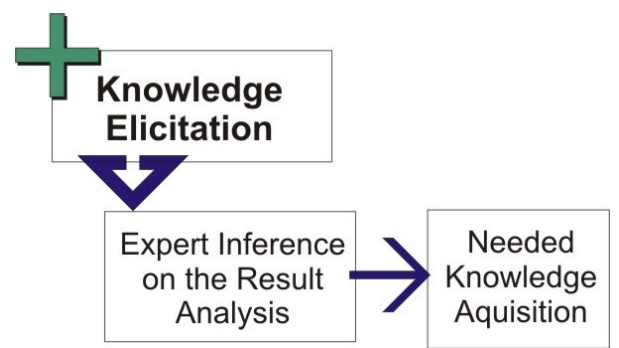

Figure 9. Inference of the expert in the Knowledge Elicitation substage

With the inference from an expert, only the necessary knowledge is acquired. This substage of PRODESEA Knowledge Base Development includes the steps six and seven from the Martin and Oxman algorithm, and the final substage implies only the step 8 from the same algorithm.

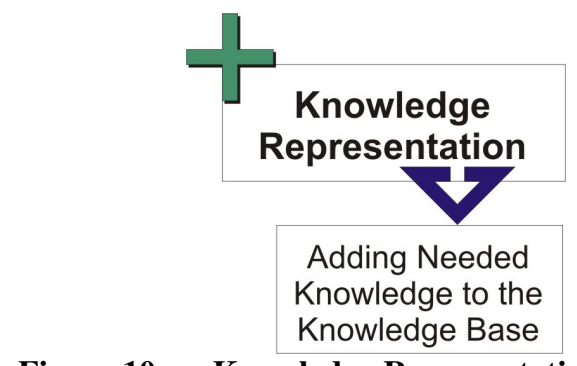

Figure 10. Knowledge Representation substage

The Knowledge Representation is not restricted to a sole technique. There are enormous variety of techniques that can be used in order to represent the knowledge, and it will suit to a knowledge engineer to choose which way will be better adapted to resolve the problem.

\section{Goal Definition}

Once the Knowledge Base achieved, a new stage comes into force. The Goal Definition must allow a list of multiple objectives to comply with. It is possible or not to attribute priorities or weights for each objective, or to

6

American Institute of Aeronautics and Astronautics 
allow the system to define this order. The important thing is to know that the Specialized System will use the acquired knowledge, which is nothing more than a set of restrictions and rules prepared during the Knowledge Base Development stage, to take decisions, like when to take a photo of determined target within a list, using for example the passage of the satellite on determined coordinates or, when it will start a satellite subsystem or when to begin a scientific experiment regarding the current load of the satellite.

PRODESEA has not yet defined a technique or strategy for this stage of acquisition for such goals. It is not necessary to mix this stage of elicitation of marks with the FOP construction. The capture of final decisions will be in charge of the planner, which using AI, will schedule the objectives to be achieved within the rules and restrictions defined by the Knowledge Base.

\section{FOP Construction}

To create a satellite plan, it is possible to develop a planner or use an already existent from the market, like for example, the APS and MVP - Adaptive Problem Solver and Multimission VICAR Planner from JPL - Jet Propulsion Laboratory, NASA or even other much simple planners like PL-PLAN, a Java Open-Source AI Planner.

What is expected from a planner at this stage of PRODESEA is to be able to build the FOP according to the material produced by the two previous stages of the process. For the INPE CCS, having the FOP automaticaly created by a planner would release considerably the human resources allocated at this time for the purpose.

In order to create a FOP in a cohesive way, the planner has to take also into account the previously created FOP for a known satellite, so it will not conflict with the satellite current missions and path, and, to have a new FOP in execution by a satellite, it must re-feed the Knowledge Base.

\section{E. FOP Test}

Before uploading a recently created FOP for an orbiting satellite to INPE CCS, it is expected that each FOP steps were previously tested by using a simulator. Isolated tests of the FOP may be carried at a first moment, to validate its cohesion and entirety, but it will never be secure if not simulated altogether with other existent or at least active FOP.

\section{F. FOP Publication}

The final stage of PRODESEA concerns the FOP delivery to be executed by the INPE CCS. Once published and scheduled for execution, the FOP stops being responsibility of PRODESEA and starts to be monitored and controlled by the CCS crew. Obviously, once published, the FOP starts to incorporate the Knowledge Base in order to be used to build new FOP.

\section{Software Products generated by the Process}

During their whole life cycle, PRODESEA documents and diagrams are generated following the modeling standard proposed by the UML. Despite the UML 2.2 supports a maximum of 14 diagrams, only 11 were proposed to be used by PRODESEA.

Structure Diagrams which were selected was the Class Diagram, Composite Structure Diagram, Deployment Diagram, Object Diagram and Package Diagram. Selected Behavior Diagrams were all choosen, Activity Diagram, State Machine Diagram and Use Case Diagram. Last, the Interaction Diagrams selected by PRODESEA were the Communication Diagram, Sequence Diagram and Timing Diagram.

The Class Diagram, which models the entities with attributes fits very well for the knowledge representation at the Knowledge Base Development stage, as well as it can be used in modeling the marks at the Goal Definition stage. The Composite Structure Diagram which describes the relations between components and classes can either be used in the knowledge representation at the Knowledge Base Development stage and tests and simulation for the FOP Test stage.

The Deployment Diagram, which treats the physical aspect of the introduction to the solution, must be employed at the FOP Publication stage and the Object Diagram, which shows the relationship between the persistence of classes, can be used at the FOP Test stage. The Package Diagram, which reflects organization, can be used to build the prototype and the knowledge representation, are both part of the Knowledge Base Development stage. Eventually, they might also be used to organize the cases of use at the Domain Definition stage.

The Activity Diagram, which demonstrates tasks flow, must be used for Business Analysis, at the Domain Definition stage, and also at the FOP Construction stage, to order the planned steps for the satellite. The State 
Machine Diagram, which demonstrates entities states changes, can be used in the FOP Construction and FOP Test stages. The Use Diagram, used for requisites capture, will be used at PRODESEA Domain Definition stage.

The Communication Diagram, which exchanges messages between objects, will be able to be used at the FOP Test stage, as well as the Sequence Diagram, which describes the tasks flow executed along the timeline. The Timing Diagram will also be used for plan tests and simulation, since this UML diagram returns the possible impact of the timeline in one or more objects, besides the timeline conditions and its effects on the objects states.

Besides diagrams, other software products will be created during the development process, like the domain problem textual description and the requisites document, both at the PRODESEA Domain Definition stage. Other documents may appear, depending on the detail level of the process steps.

\section{Conclusion}

Note that there is still much work to be done on PRODESEA. Only detailed levels for Domain Definition and Knowledge Base Development are already done, the Goal Definition, FOP Construction, FOP Test and FOP Publication stages still have to be detailed and refined.

It is expected that PRODESEA will be ready for use of automated planning by the INPE CCS at mid of 2011, and it is also expected to reach a standardized modeling process which results in resources optimizing, mostly human resources, allocated to the FOP.

\section{References}

${ }^{1}$ Neighbors, J., "Software Construction Using Components", Ph.D. Dissertation, Department of Information and Computer Science, California Univ., Irvine, CA, 1981.

${ }^{2}$ Kelly, S. and Tolvanen, J-L., Domain-Specific Modeling, Wiley-IEEE Computer Society Pr, 2008.

${ }^{3}$ Ross, J. W. et al, Enterprise Architecture As Strategy: Creating a Foundation for Business Execution, Harvard Business Press, 2006

${ }^{4}$ Nikolopoulos, C., Expert Systems: Introduction to First and Second Generation and Hybrid Knowledge Based Systems, Dekker, New York, 1997.

${ }^{5}$ Camargo, K. G., "Inteligência Artificial Aplicada à Nutrição na Prescrição de Planos Alimentares", Master Dissertation, Departamento de Engenharia de Produção e Sistemas, Universidade Federal de Santa Catarina, Trindade, SC, Brazil, 1999.

${ }^{6}$ Cordingley, E. S., "Knowledge elicitation techniques for knowledge-based systems", Knowledge elicitation: principle, techniques and applications, Springer-Verlag New York, Inc., New York, NY, 1989.

${ }^{7}$ Martin, J. and Oxman, S., Building expert systems, Engle wood Cliffs, Prentice Hall, NJ, 1988. 